\title{
Risk factors for new-onset atrial fibrillation in patients with chronic obstructive pulmonary disease: a systematic review and meta-analysis
}

\author{
Qiangru Huang ${ }^{1,2}$, Huaiyu Xiong ${ }^{1,2}$, Tiankui Shuai ${ }^{1,2}$, Meng Zhang ${ }^{1,2}$, Chuchu Zhang ${ }^{1,2}$, Yalei Wang ${ }^{1,2}$, Lei Zhu $^{1,2}$, \\ Jiaju Lu ${ }^{1,2}$, Jian Liu ${ }^{\text {Corresp. 1,2 }}$ \\ ${ }^{1}$ Department of Intensive Care Unit, The First Hospital of Lanzhou University, Lanzhou, China \\ 2 The First Clinical Medical College of the First Hospital of Lanzhou University, LanZhou, China \\ Corresponding Author: Jian Liu \\ Email address: medecinliu@sina.com
}

Background. New-onset atrial fibrillation (AF) in patients with chronic obstructive pulmonary disease (COPD) is associated with an accelerated decline in lung function, and a significant increase in mortality rate. A deeper understanding of the risk factors for new-onset AF during COPD will provide insights into the relationship between COPD and AF and guide clinical practice. This systematic review and metaanalysis is designed to identify risk factors for new-onset AF in patients with COPD, and to formulate recommendations for preventing AF in COPD patients that will assist clinical decision making.

Methods. PubMed, Embase, Web of Science and Cochrane Library databases were searched for studies, which reported the results of potential risk factors for new-onset AF in COPD patients.

Results. Twenty studies involving 8,072,043 participants were included. Fifty factors were examined as potential risk factors for new-onset AF during COPD. Risk factors were grouped according to demographics, comorbid conditions, and COPD- and cardiovascular-related factors. In quantitative analysis, cardiovascular- and demographic-related factors with a greater than $50 \%$ increase in the odds of new-onset AF included age (over 65 years and over 75 years), acute care encounter, coronary artery disease, heart failure and congestive heart failure. Only one factor is related to the reduction of odds by more than $33.3 \%$, which is black race (vs white). In qualitative analysis, the comparison of the risk factors was conducted between COPD-associated AF and non-COPD-associated AF. Cardiovascular-related factors for non-COPD-associated AF were also considered as risk factors for new-onset AF during COPD; however, the influence tended to be stronger during COPD. In addition, comorbid factors identified in non-COPD-associated AF were not associated with an increased risk of AF during COPD.

Conclusions. New-onset AF in COPD has significant demographic characteristics. Older age (over 65 years), males and white race are at higher risk of developing AF. COPD patients with a history of cardiovascular disease should be carefully monitored for new-onset of AF, and appropriate preventive measures should be implemented. Even patients with mild COPD are at high risk of new-onset AF. This study shows that risk factors for new-onset AF during COPD are mainly those associated with the cardiovascular-related event and are not synonymous with comorbid factors for non-COPD-associated AF. The pathogenesis of COPD-associated AF may be predominantly related to the cardiac dysfunction caused by the chronic duration of COPD, which increases the risk of cardiovascular-related factors and further increases the risk of AF during COPD.

PROSPERO registration number. CRD42019137758.

Peer) reviewing PDF | (2020:02:46024:4:0:NEW 3 Oct 2020) 


\section{Risk factors for new-onset atrial fibrillation in patients}

2 with chronic obstructive pulmonary disease: a

3 systematic review and meta-analysis

4

5 Qiangru Huang ${ }^{1,2}$, Huaiyu Xiong ${ }^{1,2}$, Tiankui Shuai ${ }^{1,2}$, Meng Zhang ${ }^{1,2}$, Chuchu Zhang ${ }^{1,2}$, Yalei

6 Wang ${ }^{1,2}$, Lei Zhu ${ }^{1,2}$, Jiaju Lu ${ }^{1,2}$, Jian Liu ${ }^{1,2}$ *

7

8 1. Department of Intensive Care Unit, The First Hospital of Lanzhou University, Lanzhou, China;

9 2. The First Clinical Medical College of the First Hospital of Lanzhou University, Lanzhou, 10 China;

11 Corresponding Author:

12 Jian Liu ${ }^{1,2}$

13 The First Hospital of Lanzhou University, Lanzhou, China.

14 Email address: medecinliu@sina.com 


\section{Abstract}

36

37

38

39

40

41

42

43

44

45

46

47

Background. New-onset atrial fibrillation (AF) in patients with chronic obstructive pulmonary disease (COPD) is associated with an accelerated decline in lung function, and a significant increase in mortality rate. A deeper understanding of the risk factors for new-onset AF in COPD patients will provide insights into the relationship between COPD and AF and guide clinical practice. This systematic review and meta-analysis is designed to identify risk factors for newonset AF in patients with COPD, and to formulate recommendations for preventing AF in COPD patients that will assist clinical decision making.

Methods. PubMed, Embase, Web of Science and Cochrane Library databases were searched for studies, which reported the results of potential risk factors for new-onset AF in COPD patients.

Results. Twenty studies involving 8,072,043 participants were included. Fifty factors were examined as potential risk factors for new-onset AF in COPD patients. Risk factors were grouped according to demographics, comorbid conditions, and COPD- and cardiovascularrelated factors. In quantitative analysis, cardiovascular- and demographic-related factors with a greater than $50 \%$ increase in the odds of new-onset AF included age (over 65 years and over 75 years), acute care encounter, coronary artery disease, heart failure and congestive heart failure. Only one factor is related to the reduction of odds by more than $33.3 \%$, which is black race (vs white). In qualitative analysis, the comparison of the risk factors was conducted between COPDassociated AF and non-COPD-associated AF. Cardiovascular-related factors for non-COPDassociated AF were also considered as risk factors for new-onset AF in COPD patients; however, 
55 the influence tended to be stronger in COPD patients. In addition, comorbid factors identified in

56 non-COPD-associated AF were not associated with an increased risk of AF in COPD patients.

57 Conclusions. New-onset AF in COPD has significant demographic characteristics. Older age

58 (over 65 years), males and white race are at higher risk of developing AF. COPD patients with a

59 history of cardiovascular disease should be carefully monitored for new-onset of AF, and

60 appropriate preventive measures should be implemented. Even patients with mild COPD are at

61 high risk of new-onset AF. This study shows that risk factors for new-onset AF in COPD

62 patients are mainly those associated with the cardiovascular-related event and are not

63 synonymous with comorbid factors for non-COPD-associated AF. The pathogenesis of COPD-

64 associated AF may be predominantly related to the cardiac dysfunction caused by the chronic

65 duration of COPD, which increases the risk of cardiovascular-related factors and further

66 increases the risk of AF in COPD patients.

67 PROSPERO registration number. CRD42019137758

Introduction 

mortality worldwide (2018b), and is predicted to rank third by 2030 (Adeloye et al. 2015). Atrial

77 fibrillation (AF) often has a negative impact on patients with chronic disease, and is the most common arrhythmia in patients with COPD (2018a). The co-existence of COPD and AF is associated with mutual exacerbation of the conditions, which complicates the management in clinical practice (Gu et al. 2013). AF in patients with COPD is associated with an accelerated decline in lung function, and a significant increase in cardiovascular accidents and long-term mortality (Méndez-Bailón et al. 2017). Reduced lung function also increases the risk of newonset AF. In a prospective cohort study, the risk of new-onset AF was 1.8-times higher for forced expiratory volume in one second between $60 \%-80 \%$ (i.e., $60 \% \leq \mathrm{FEV}_{1}<80 \%$ ) of predicted compared with $\mathrm{FEV}_{1} \geq 80 \%$. In addition, the risk of AF related hospitalization was 1.3 times higher in $60 \% \leq \mathrm{FEV}_{1}<80 \%$ of predicted and 1.8 -times higher in $\mathrm{FEV}_{1}<60 \%$ of predicted compared with $\mathrm{FEV}_{1} \geq 80 \%$ of predicted (Buch et al. 2003). $\mathrm{AF}$ is combined with an alarming mortality rate. The incidence of AF in severe COPD patients is approximately four times higher than that in non-COPD patients (Konecny et al. 2014). The inhospital mortality rate for patients with COPD-associated AF is $2.9 \%$, whereas the corresponding rate for non-COPD-associated AF patients is 2.2\% (Méndez-Bailón et al. 2017). Although the prevalence and short-term mortality rate of new-onset AF in COPD patients are high, the management of cardiovascular conditions in COPD patients is often ignored in current clinical practice. Elucidating the risk factors will provide an effective approach to screening high-risk 
96 COPD patients with new-onset AF and improving the clinical course and prognosis of COPD. A

97 deeper understanding of the risk factors for new-onset AF in COPD patients will also provide insights into the relationship between COPD and AF and guide clinical practice. Based on the mutual promotion of deterioration between COPD and AF and the difference in primary disease settings, the risk factors for AF in the non-COPD setting do not adequately reflect the risk of AF in COPD patients. However, there is a paucity of information on the incidence of AF and insufficient evidence of risk factors in COPD patients from most studies.

Comprehensive integration of risk factors for $\mathrm{AF}$ in patients with $\mathrm{COPD}$ is important in bridging the knowledge gaps and providing information for the application of COPD guidelines. Therefore, this systematic review and meta-analysis was designed to identify risk factors for new-onset AF in patients with COPD, and to formulate recommendations for limiting AF in COPD patients that will assist clinical decision making.

\section{Materials \& Methods}

All methods of this systematic reviews and meta-analyses followed the Preferred Reporting Items for Systematic Reviews and Meta-Analyses (PRISMA) guidelines (Wang et al. 2018a). Details of the preregistered protocol for this study are available on International prospective register of systematic reviews (PROSPERO; CRD42019137758).

\section{Data sources and searches}

The review authors searched for medical literature before December 2019. The search was conducted in four electronic databases including the Cochrane Library, PubMed, Embase, Web 
117 of Science (WOS), and the reference lists from review articles. The search strategy used a

118 combination of MeSH, Emtree and text word, conducted with the following keywords: COPD or

119 chronic obstructive pulmonary disease and AF or atrial fibrillation. The review articles with

120 relevant topic were screened through keyword search, and their reference lists were further

121 searched for eligible studies. The details of search strategies can be found in the Supplementary

122 (Appendix 1. Search Strategy).

123 This meta-analysis included studies that met the following inclusion criteria:

124 1. Adult patients diagnosed with COPD (18 years of age or older). COPD was diagnosed

125 according to the latest reference standards during the study, such as the GOLD criteria.

1262 . The studies reported the results of identified risk factors for new-onset AF in COPD

127 patients. The adjusted odds ratio (OR), SE, and 95\% confidence intervals (CI) for each

128 candidate risk factor are reported as appropriate.

129 3. No publication date, status or language restrictions were applied.

$130 \quad$ Exclusion criteria:

$131 \quad$ 1. Secondary study, editorials, and animal experiments were excluded.

2. Studies that reported ORs with $95 \%$ CIs and $P$ values showed inconsistency were excluded.

3. Studies that included only unadjusted OR and studies that involved new-onset AF in patients only after cardiothoracic surgery were excluded.

Study selection 
138

139

140

141

142

143

144

145

146

147

148

149

150

151

152

153

154

155

156

157

of the titles, abstracts and keywords. If a study was found relevant to this topic, the full text

would be further evaluated by at least two reviewers to assess whether it fulfills the selection

criteria. In case of disagreement between the reviewers, the third reviewer ( $\mathrm{J}$ Liu) would be

consulted to resolve the disagreement. An inter-rater reliability analysis was conducted to assess

the consistency of the study selection between two independent review authors. A study diagram was prepared for this selection to demonstrate the entire process of literature research and the selection of studies.

\section{Data extraction and quality assessment}

The data was independently extracted by two review authors (Q Huang and H Xiong), and the resulting differences were resolved by a third reviewer (J Liu). The extracted data included: 1) citation information, participant characteristics, study types, and study settings; 2) definitions of COPD and AF; and 3) the adjusted OR, SE, and 95\% CI for each candidate risk factor. Note that none of the studies from which data was extracted include more than one model where multiple variables were included, and any included study using empirical model building strategies (such as forward selection, stepwise approaches or backward elimination) may exclude variables from the adjusted models. If the data were insufficient, an email was sent to the authors to request the data. Note that all included studies provide complete information on the main outcomes.

Two review authors ( $\mathrm{L}$ Liu and $\mathrm{J} \mathrm{Lu}$ ) independently applied the guidelines of the PRISMA statement to evaluate each involved study (Liberati et al. 2009). For observational studies such as 
158

159

160

161

162

163

164

165

166

167

168

169

170

171

172

173

174

175

176

177

case-control and cohort studies, the Newcastle-Ottawa Scale (NOS) was used (Lo et al. 2014) to assess the methodological quality and risk of bias (Table S1 and Table S2). In case of any inconsistency, an agreement was reached through discussion between all authors.

\section{Data synthesis and risk factor analysis}

Extracted data were analyzed using Stata SE 14.0 (Stata Corp; College Station, TX, USA).

Considering the play of chance and some genuine variation in risk-factor effects, the pooled effect size was calculated by the random-effects method in this study. The heterogeneity of eligible studies was assessed by the Cochrane $Q$ test (substantive heterogeneity was indicated by $P<0.05$ ) and the $I^{2}$ test (substantive heterogeneity was indicated by $I^{2}>50 \%$ ). If substantive heterogeneity existed, sensitivity analysis was performed to analyze the potential sources of the heterogeneity. Egger's test was used for assessing the publication bias in case that there are over 10 included studies (Egger et al. 1997). The $\alpha$ value was set to 0.05 .

Risk factors were grouped by factor type (demographic, comorbid condition, COPD-related factors and cardiovascular-related factors), and stratified by effect size, with specific consideration for factors associated with a greater than $50 \%$ increase or $33.3 \%$ decrease in odds of new-onset AF (the inverse of a $50 \%$ increase in odds corresponds to a $33.3 \%$ decrease in odds).

95\% CIs were used to estimate the precision of the ORs. A wide CI indicates lower OR precision, whereas a narrow CI indicates higher OR precision. If a 95\% CI spans 1.0, the increased or decreased odds does not reach statistical significance. Usually $95 \%$ CI excludes null 
178 value means $P<0.05$. The $95 \%$ CI and $P$ value of potential risk factors involved in this study

179 showed consistency.

180

181 Quantitative analysis

182 Quantitative meta-analysis examined candidate risk factors identified in at least two studies

183 (Jackson et al. 2018). The primary result was a pooled-adjusted OR for each risk factor using a

184 general inverse variance method with random effects model. When the source publication is

185 unavailable, SEs were calculated using the following formula:

186

$S E=\frac{\ln (\text { Upper } \quad C I)-\ln (\text { Lower } \quad C I)}{3.92}$

187

188

189

190

191

192

193

194

195

and the general public were not involved in this study.

Qualitative analysis and Schnabel et al (Schnabel et al. 2009).

\section{Patient and public involvement}

196

197

All included risk factors (pooled and un-pooled) were reported in at least one study. To assess whether increased risk factors for community-associated AF also increased the risk of COPD-associated AF, we compared the risk factors of new-onset AF in COPD with those of community-associated AF previously identified in Chamberlain et al. (Chamberlain et al. 2011)

This study is a meta-analysis using data from previously published studies, hence patients

Results

\section{Study selection and study characteristics}


199

200

201

202

203

204

205

206

207

208

209

210

211

212

213

214

215

216

217

218

219

A flow chart of the study selection process prepared according to the PRISMA guidelines is presented in Figure 1 (Lo et al. 2014). After reviewing the title and abstract, 150 articles were screened for full-text review. Of these, 130 articles failed to meet the inclusion criteria. Twenty studies were found to fulfill all the criteria and involved 8,072,043 participants. Inter-rater reliability was strong $(\mathrm{K}, 0.80)$. Of the 20 included studies, 13 studies used retrospective cohort designs (Alves Guimaraes et al. 2018; Chen \& Liao 2018; Desai et al. 2019; Ganga et al. 2013;

Genao et al. 2015; Hu \& Lin 2018; Koskela et al. 2014; Lainscak et al. 2009; Liao \& Chen 2017;

Rusinowicz et al. 2017; Wang et al. 2018b; Warnier et al. 2010; Xiao et al. 2019a), three were

prospective cohorts (Hirayama et al. 2018; Short et al. 2012; Volchkova et al. 2015), three were case-control studies (Celli et al. 2010; Nadeem et al. 2015; Tomioka et al. 2019), and one was a prospective nested case-control study (Wilchesky et al. 2012). All studies examined new-onset AF in patients with COPD. A summary of the characteristics of the included studies is shown in Table 1.

\section{Methodological quality and risk of bias}

As shown in Table 1, the methodological quality of the observational studies was rated high, with 10 studies scoring nine of nine on the NOS (Alves Guimaraes et al. 2018; Celli et al. 2010; Chen \& Liao 2018a; Desai et al. 2019; Genao et al. 2015; Short et al. 2012; Tomioka et al. 2019; Wang et al. 2018b; Warnier et al. 2010; Xiao et al. 2019b), six scoring eight of nine (Hirayama et al. 2018; Hu \& Lin 2018; Koskela et al. 2014; Lainscak et al. 2009; Nadeem et al. 2015; Volchkova et al. 2015), three scoring seven of nine (Ganga et al. 2013; Liao \& Chen 2017; Wilchesky et al. 2012), and one scoring six of nine (Rusinowicz et al. 2017). The overall risk of 
220

221

222 (Supplementary file; Appendix 2).

bias was rated low; however, the included studies used different disease definitions, which influenced the population selection. Summaries of the risk of bias are shown in Tables S1 and S2

223

224

225

226

227

228

229

230

231

232

233

234 235

236

237

238

239

\section{Risk factor analysis}

\section{Quantitative analysis}

Among all risk factors, the $95 \% \mathrm{CI}$ and $P$ value of pooled OR showed consistency. Figure 2 shows the effect sizes of all 50 factors (un-pooled and pooled) examined as potential risk factors for new-onset AF in COPD patients. Twenty-five factors presented in at least two studies were included in the pooled meta-analysis. Seventeen pooled factors were associated with increased odds of new-onset AF: age (over 65 years and over 75 years), male, urban population, any acute care encounter, sepsis, renal failure, pneumonia, acute respiratory failure, invasive mechanical ventilation (IMV), noninvasive mechanical ventilation (NMV), ipratropium bromide use, shortacting $\beta$-agonist use, myocardial infarction (MI), coronary artery disease (CAD), heart failure (HF) and congestive heart failure (CHF). Of these, six factors were associated with a greater than $50 \%$ increase in odds: age (over 65 years and over 75 years), acute care encounter, CAD, HF and CHF.

Two pooled factors were associated with decreased odds of new-onset AF in the metaanalysis: black ethnicity (compared with white), and peripheral vascular disease (PVD). Only one factor is related to the reduction of odds by more than $33.3 \%$, which is black race (vs white).

Six factors were not associated with new-onset AF after the pooled analysis: hypertension, 
240 hyperlipidemia, diabetes mellitus (DM), hepatic failure, long-acting beta-agonist use and

241 theophylline use.

242 Heterogeneity

243 Heterogeneity analysis showed that $I^{2}$ was greater than $50 \%$ in nine pooled risk factors,

244 seven of which were included the study by Desai et al. (Desai et al. 2019). The direction of the

245 effects in individual study ORs from five of these seven pooled factors included in the study by

246 Desai et al. (Desai et al. 2019) was concordant (black ethnicity, hepatic failure, IMV, MI and

247 CAD). The direction of the effect estimates in individual ORs from the other two pooled risk

248 factors (hypertension and acute respiratory failure) was discordant. The other two pooled factors

249 with high heterogeneity were not included in the study by Desai et al. (Desai et al. 2019) (acute

250 care and NMV) and both included only two studies that reported the risk factor and had the same

251 direction of effect for individual study ORs.

252 Sensitivity analysis of acute respiratory failure, MI and CAD showed that the high

253 heterogeneity was derived from the study by Desai et al. (Desai et al. 2019). The results of

254 sensitivity analysis are shown in Supplementary Figures S1, S2, and S3 (Appendix 3. Sensitivity

255 analysis). When the study by Desai et al. (Desai et al. 2019) was excluded from the analysis, $I^{2}$

256 decreased from $57 \%$ to $0 \%$ and the OR decreased from 1.13 (95\% CI: $1.12-1.15)$ to 1.09 (95\%

257 CI: 1.06-1.12) for acute respiratory failure, $I^{2}$ decreased from $98 \%$ to $63 \%$ and the OR increased

258 from 1.41 (95\% CI: 1.19-1.69) to 1.55 (95\% CI: 1.29-1.85) for MI and $I^{2}$ decreased from $94 \%$

259 to $35 \%$ and the OR increased from 2.57 (95\% CI: $1.01-6.49)$ to 3.88 (95\% CI: $2.43-6.21)$ for

260 CAD.

Peer) reviewing PDF | (2020:02:46024:4:0:NEW 3 Oct 2020) 
261

262

263

264

265

266

267

268

269

270

271

272

273

274

275

276

277

278

279

280

281

\section{Qualitative analysis}

All of the un-pooled ORs showed the $95 \% \mathrm{CI}$ and $P$ value with consistency. To

qualitatively compare the risk factors of COPD-associated AF and community-associated AF

risk factors, we included 25 additional factors that were evaluated in only one study. Eleven un-

pooled factors were associated with a greater than $50 \%$ increase in odds of new-onset AF,

including lower health-related quality of life (HRQoL; HRQoL was assessed using the self-

administered Airways Questionnaire 20 (AQ20). Low HRQoL is defined as AQ20 summary

score $\geq 14$, chronic kidney disease (CKD), vascular disease, thyroid diseases, worsening MRC

dyspnea score, history of cardiac arrest, valvular heart disease, history of cardiogenic shock,

cardiomyopathy, left ventricular hypertrophy, and obstructive sleep apnea (OSA). No factor was

associated with a greater than $33.3 \%$ decrease in the odds. Eight factors were not associated with

new-onset $\mathrm{AF}$ in terms of odds, including cancer, neurologic dysfunction, hematologic

dysfunction, metabolic dysfunction, prior beta-blocker use, tiotropium use, betamimetics and ipratropium use and venous thromboembolism.

Of the 11 community-associated factors identified by Schnabel et al. (Schnabel et al. 2009)

and the 20 community-associated factors identified by Chamberlain et al. (Chamberlain et al.

2011), 10 factors had similar definitions compared with COPD-related factors evaluated in the

current study, which allowed a direct comparison. A summary of the comparison of the effect

sizes of risk factors for COPD-associated versus community-associated AF is shown in Table 2.

In general, comorbid conditions associated with increased risk for community-associated AF

(e.g., DM, prior beta-blocker use, and hypertension) were not associated with higher risk of new-

Peer) reviewing PDF | (2020:02:46024:4:0:NEW 3 Oct 2020) 
282

283

284

285

286

287

288

289

290

291

292

293

294

295

296

297

298

299

300

301

302

onset AF in COPD patients. Cardiovascular-related factors for community-associated AF were also identified as risk factors for new-onset AF in COPD patients, and the effective estimates tended to be stronger in COPD patients.

\section{Discussion}

In this, study, we conducted a systematic review and meta-analysis to characterize and evaluate risk factors for new-onset AF in COPD patients. Fifty potential risk factors were grouped by factor type. Quantitative analysis demonstrated that risk factors for new-onset AF in COPD patients are mainly those associated with cardiovascular-related events and demographic factors (e.g. age over 65, age over 75, acute care encounter, CAD, HF and CHF). Although COPD-related factors can also trigger new-onset AF in COPD patients, the risk effect is moderate. In this study, we further explored the differences between the risk factors for COPDassociated AF and the identified risk factors for non-COPD-associated AF. Although cardiovascular-related factors for non-COPD-associated AF are also considered to be risk factors for new-onset $\mathrm{AF}$ in COPD patients, the influences are stronger in COPD patients. Interestingly, comorbid factors (DM, prior beta-blocker use, hypertension, PVD and hepatic failure) identified in non-COPD-associated AF are not associated with an increased risk of new-onset $\mathrm{AF}$ in $\mathrm{COPD}$ patients. This highlights the importance of distinguishing risk factors for AF in patients with COPD and the potential need for different preventive interventions.

New-onset AF in COPD patients has significant demographic characteristics. Older age (over 65 years and over 75 years), male and whites are at higher risk of morbidity. Among all the 
303 demographic factors, older age is the dominant factor (over 65 years: $\mathrm{OR}=3.29$; over 75 years:

$304 \mathrm{OR}=4.51$ ). Both COPD and AF have age distribution characteristics, and the prevalence

305 increases with age. In the study conducted by Méndez-Bailón et al., they found that the

306 prevalence in AF patients with COPD also showed a increasing tendency with age. Their results

307 indicate that the overall prevalence of COPD was approximately $17 \%$ in AF patients, increasing

308 to more than $40 \%$ in individuals aged $>75$ years. (Méndez-Bailón et al. 2017). This information

309 highlights demographic characteristics that can be used by clinicians to pre-screen high-risk

310 populations.

311 The most commonly identified risk factors for new-onset AF in COPD patients are

312 associated with cardiovascular events. Although these factors are also considered to be risk

313 factors for non-COPD-related AF, the risk of cardiovascular events in patients with COPD is

314 often amplified compared to that in non-COPD patients. As a chronic disease, COPD is

315 independently associated with cardiovascular risk (Sidney et al. 2005). The adverse effects of

316 COPD on the heart are multifaceted. This condition not only exacerbates organic heart disease

317 and directly induces AF, but also induces hypoxia, hypercapnia (Ferraro et al. 2019), electrolyte

318 disturbances and increased blood viscosity, which exacerbate the cardiac burden and oxygen

319 consumption, and may therefore lead to deterioration of cardiac function in the presence of

320 concomitant cardiovascular disease (Feary et al. 2010; Fuster et al. 2006). Furthermore,

321 sympathetic nerve overactivity and the aggravation of COPD-associated inflammation,

322 especially in acute exacerbation of COPD, can lead to increased heart rate and cardiac events

323 (Andreas et al. 2005; Lopez \& House-Fancher 2005). Due to the mutual exacerbation of COPD 
324 and cardiovascular disease, the risk of cardiovascular-related factors is more notable in patients 325 with COPD.

326 In the chronic process, patients with chronic COPD have a higher risk of cardiac

327 abnormalities (including HF, CAD and MI) than non-COPD patients. These organic

328 cardiopathies contribute to the occurrence of AF. The results of this study demonstrate a

329 potential association between cardiovascular-related history and new-onset AF in patients with

330 COPD. However, in clinical practice, clinicians often ignore the history of heart disease in

331 patients with COPD (Konecny et al. 2014). Attention to the cardiac history of COPD patients can

332 help with early management and clinical decision-making regarding the necessary interventions,

333 thereby effectively reducing the risk of new-onset AF in COPD patients.

334 Although the risk effect of COPD-related factors is not as strong as cardiovascular and

335 demographic factors, the impact on COPD-associated AF remains substantial. Moreover, we

336 found that slight decreases in the pulmonary function of COPD patients were associated with

337 increased risk of AF; therefore, this situation requires careful monitoring and prevention. As a

338 remedy for NMV failure, IMV usually indicates a further deterioration of pulmonary function in

339 COPD patients. We found that not only IMV, but also NMV, risk triggering AF. This finding is

340 consistent with the study by Buch et al. (Buch et al. 2003), which showed that the risk of

341 triggering new-onset $\mathrm{AF}$ was 1.8 -times higher for $60 \% \leq \mathrm{FEV}_{1}<80 \%$ of predicted compared

342 with $\mathrm{FEV}_{1} \geq 80 \%$.

343 In addition, we found that COPD-related factors have the potential to trigger AF (e.g.

344 pneumonia, acute respiratory failure). In accordance with our findings, previous studies have 
345 shown that new-onset AF in COPD patients can be caused by COPD-related factors, such as the

346 inflammatory state of COPD and the use of beta-agonists. In animal models, streptococcus-

347 induced pneumonia has been shown to cause myocardial micro-abscesses, which can develop

348 into fibrotic lesions prone to arrhythmia formation (Ganga et al. 2013). The formation of

349 arrhythmic lesions may explain our findings of increased risk of AF in patients with pneumonia.

350 Comorbid conditions are major factors that can be used to predict the risk of community-

351 associated AF. However, our findings suggest that the risk effects of comorbid conditions are

352 low compared with the effects of cardiovascular-related or COPD-related factors. This may

353 reflect the greater severity of the disease (e.g. HF, CAD and MI) in COPD patients or fewer

354 competing risk factors, resulting in a lower proportion of comorbid factors attributed to new-

355 onset AF in patients with COPD (Chamberlain et al. 2011; Vestbo et al. 2013). Compared with

356 other comorbid conditions, COPD itself is the greater risk for triggering AF.

357 This study of risk factors contributed to our understanding of the pathogenesis of AF in

358 COPD patients. AF is thought to occur in two steps that involve the production of

359 arrhythmogenic substrates (Liu et al. 2017), followed by the triggering event (Aldhoon et al.

360 2010). In this analysis, we identified chronic factors that are known to potentiate the formation of

361 atrial fibrosis following long-term exposure (e.g. CHF and CAD), which deteriorate further in

362 the setting of COPD. We also identified several COPD-related factors that may trigger AF (e.g.

363 pneumonia, acute respiratory failure, IMV and NMV). However, the risk effect of cardiovascular

364 factors was found to be greater than that of COPD-related factors. This suggests that new-onset 
365 AF in patients with COPD is caused mainly by the deterioration in cardiac function during

366 chronic COPD, with the acute deterioration of COPD itself affecting the occurrence of AF.

367 There are several limitations in the current systematic review and meta-analysis. Firstly,

368 there was substantial heterogeneity in the results of some risk factors. In order to respond to this

369 limitation, we then performed a sensitivity analysis to explore the potential sources of

370 heterogeneity. We identified the study by Desai et al. (Desai et al. 2019) as the major source of

371 heterogeneity in three risk factors, including acute respiratory failure ( $I^{2}$ decreased from $57 \%$ to

$3720 \%)$, MI ( $I^{2}$ decreased from $98 \%$ to $\left.63 \%\right)$ and CAD ( $I^{2}$ decreased from $94 \%$ to $\left.35 \%\right)$. The

373 analysis of heterogeneity is limited due to the insufficient number of studies reporting risk

374 factors. In addition, other potential sources of heterogeneity in our analysis of the results of

375 baseline characteristics are as follows: 1) The designs of the included studies were

376 heterogeneous. Compared with meta-analyses including randomized controlled trials, those

377 including observational studies have greater potential bias. 2) The studies included patients with

378 diseases defined using different strategies. Although all strategies are reasonable, different

379 criteria may lead to variations in clinicians' decision-making, which may increase the

380 heterogeneity of population selection.

381 Secondly, interpretation of the observed ORs depends on all necessary confounders being

382 included, without any colliders or intermediate variables. However, the adjusted ORs reported in

383 the included studies cannot eliminate residual confounding caused by unknown, missed and

384 inaccurate confounding factors, and there is a lack of discussion about the causality of the

385 variables. Therefore, limited by the existing methods, it is difficult to analyze the causal 
386 associations of 50 variables without additional original research. Furthermore, we also suggest

387 more original research to use the causal modelling approaches such as directed acyclic graphs to

388 distinguish between confounders, colliders, and intermediate variables when considering causal

389 risk factors. Thirdly, due to different research purposes, the included studies only reported the

390 results of some potential risk factors related to the research purpose, while other variables were

391 only mentioned as an adjusted variable, and did not report the adjusted ORs and 95\% CIs. Thus,

392 the number of risk factors included in the meta-analysis was less than the number of adjusted

393 variables included in the adjusted models. This suggests that more original studies are needed to

394 report more comprehensive results for further analysis and evaluation of specific risk factors. In

395 addition, because no risk factor was reported in 10 or more original studies; Egger's test and

396 funnel plots were not included in our analysis. Further high-quality original studies can also help

397 to limit the potential publication bias.

398

399 Conclusions

400 This systematic review and meta-analysis reveals the demographic characteristics of

401 patients with new-onset AF in COPD patients. Older age (over 65 years and over 75 years),

402 males and whites are at higher risk of developing AF. The dominant factors are cardiovascular

403 (CAD, HF and CHF), and may be amplified in the context of COPD. COPD patients with a

404 history of cardiovascular disease should be carefully monitored for new-onset AF, and

405 appropriate preventive measure should be implemented. Even for patients with mild COPD,

406 clinicians should not relax their vigilance in monitoring patients for new-onset AF. The

Peer) reviewing PDF | (2020:02:46024:4:0:NEW 3 Oct 2020) 
407 pathogenesis of AF in COPD patients may be primarily related to cardiac dysfunction caused by

408 the chronic duration of COPD, which increases the risk effect of cardiovascular-related factors

409 and further increases the risk of AF in patients with COPD. This highlights the importance of

410 identifying risk factors for predicting COPD-associated AF and the potential need for different

411 preventive interventions.

412

\section{Acknowledgements}

414 The authors gratefully acknowledge the support of the First Clinical Hospital of Lanzhou 415 University, the first clinical medical college of Lanzhou University, Evidence-based Medicine 416 Center of Lanzhou University, and all the authors who participated in this study.

417

\section{References}

419

420

421

422

423

424

425

426

427

428

429

430

431

432

433

434

435

436

437

438

2018a. Corrigendum to: 2016 ESC Guidelines for the management of atrial fibrillation developed in collaboration with EACTS. Eur Heart J 39:1109. 10.1093/eurheartj/ehx039

2018b. Global, regional, and national age-sex-specific mortality and life expectancy, 1950-2017: a systematic analysis for the Global Burden of Disease Study 2017. Lancet 392:1684-1735. 10.1016/s0140-6736(18)31891-9

Adeloye D, Chua S, Lee C, Basquill C, Papana A, Theodoratou E, Nair H, Gasevic D, Sridhar D, Campbell H, Chan KY, Sheikh A, and Rudan I. 2015. Global and regional estimates of COPD prevalence: Systematic review and meta-analysis. J Glob Health 5:020415. 10.7189/jogh.05-020415

Aldhoon B, Melenovský V, Peichl P, and Kautzner J. 2010. New insights into mechanisms of atrial fibrillation. Physiol Res 59:1-12.

Alves Guimaraes JP, Goncalves F, Moz M, Mateus P, Trigo J, and Moreira JI. 2018. Chronic obstructive pulmonary disease in acute coronary syndrome patients and its impact on prognosis. Eur Heart J 39:1350. 10.1093/eurheartj/ehy566.P6409

Andreas S, Anker SD, Scanlon PD, and Somers VK. 2005. Neurohumoral activation as a link to systemic manifestations of chronic lung disease. Chest 128:3618-3624. 10.1378/chest.128.5.3618

Buch P, Friberg J, Scharling H, Lange P, and Prescott E. 2003. Reduced lung function and risk of atrial fibrillation in the Copenhagen City Heart Study. Eur Respir J 21:1012-1016. 10.1183/09031936.03.00051502

Celli B, Decramer M, Leimer I, Vogel U, Kesten S, and Tashkin DP. 2010. Cardiovascular safety of tiotropium in patients with COPD. Chest 137:20-30. 10.1378/chest.09-0011

Chamberlain AM, Agarwal SK, Folsom AR, Soliman EZ, Chambless LE, Crow R, Ambrose M, and Alonso A. 2011. A clinical risk score for atrial fibrillation in a biracial prospective cohort (from the Atherosclerosis Risk in

Peer) reviewing PDF | (2020:02:46024:4:0:NEW 3 Oct 2020) 
439

440

441

442

443

444

445

446

447

448

449

450

451

452

453

454

455

456

457

458

459

460

461

462

463

464

465

466

467

468

469

470

471

472

473

474

475

476

477

478

479

Communities [ARIC] study). Am J Cardiol 107:85-91. 10.1016/j.amjcard.2010.08.049

Chen CY, and Liao KM. 2018a. The impact of atrial fibrillation in patients with COPD during hospitalization. Int J Chron Obstruct Pulmon Dis 13:2105-2112. 10.2147/copd.S166534

Desai R, Patel U, Singh S, Bhuva R, Fong HK, Nunna P, Zalavadia D, Dave H, Savani S, and Doshi R. 2019. The burden and impact of arrhythmia in chronic obstructive pulmonary disease: Insights from the National Inpatient Sample. Int J Cardiol 281:49-55. 10.1016/j.ijcard.2019.01.074

Egger M, Davey Smith G, Schneider M, and Minder C. 1997. Bias in meta-analysis detected by a simple, graphical test. Bmj 315:629-634. 10.1136/bmj.315.7109.629

Feary JR, Rodrigues LC, Smith CJ, Hubbard RB, and Gibson JE. 2010. Prevalence of major comorbidities in subjects with COPD and incidence of myocardial infarction and stroke: a comprehensive analysis using data from primary care. Thorax 65:956-962. 10.1136/thx.2009.128082

Ferraro M, Di Vincenzo S, Dino P, Bucchieri S, Cipollina C, Gjomarkaj M, and Pace E. 2019. Budesonide, Aclidinium and Formoterol in combination limit inflammaging processes in bronchial epithelial cells exposed to cigarette smoke. Exp Gerontol 118:78-87. 10.1016/j.exger.2019.01.016

Fuster V, Rydén LE, Cannom DS, Crijns HJ, Curtis AB, Ellenbogen KA, Halperin JL, Le Heuzey JY, Kay GN, Lowe JE, Olsson SB, Prystowsky EN, Tamargo JL, Wann S, Smith SC, Jr., Jacobs AK, Adams CD, Anderson JL, Antman EM, Hunt SA, Nishimura R, Ornato JP, Page RL, Riegel B, Priori SG, Blanc JJ, Budaj A, Camm AJ, Dean V, Deckers JW, Despres C, Dickstein K, Lekakis J, McGregor K, Metra M, Morais J, Osterspey A, and Zamorano JL. 2006. ACC/AHA/ESC 2006 guidelines for the management of patients with atrial fibrillation--executive summary: a report of the American College of Cardiology/American Heart Association Task Force on Practice Guidelines and the European Society of Cardiology Committee for Practice Guidelines (Writing Committee to Revise the 2001 Guidelines for the Management of Patients With Atrial Fibrillation). J Am Coll Cardiol 48:854-906. 10.1016/j.jacc.2006.07.009

Ganga HV, Nair SU, Puppala VK, and Miller WL. 2013. Risk of new-onset atrial fibrillation in elderly patients with the overlap syndrome: a retrospective cohort study. J Geriatr Cardiol 10:129-134. 10.3969/j.issn.16715411.2013.02.001

Genao L, Durheim MT, Mi X, Todd JL, Whitson HE, and Curtis LH. 2015. Early and long-term outcomes of older adults after acute care encounters for chronic obstructive pulmonary disease exacerbation. Annals of the American Thoracic Society 12:1805-1812. 10.1513/AnnalsATS.201504-2500C

Gu J, Liu X, Tan H, Zhou L, Jiang W, Wang Y, Liu Y, and Gu J. 2013. Impact of chronic obstructive pulmonary disease on procedural outcomes and quality of life in patients with atrial fibrillation undergoing catheter ablation. J Cardiovasc Electrophysiol 24:148-154. 10.1111/j.1540-8167.2012.02448.x

Hirayama A, Goto T, Shimada YJ, Faridi MK, Camargo CA, Jr., and Hasegawa K. 2018. Acute Exacerbation of Chronic Obstructive Pulmonary Disease and Subsequent Risk of Emergency Department Visits and Hospitalizations for Atrial Fibrillation. Circ Arrhythm Electrophysiol 11:e006322. 10.1161/circep.118.006322

Hu WS, and Lin CL. 2018. CHA2DS2-VASc score for ischaemic stroke risk stratification in patients with chronic obstructive pulmonary disease with and without atrial fibrillation: a nationwide cohort study. Europace 20:575-581. 10.1093/europace/eux065

Jackson D, Law M, Stijnen T, Viechtbauer W, and White IR. 2018. A comparison of seven random-effects models for meta-analyses that estimate the summary odds ratio. Stat Med 37:1059-1085. 10.1002/sim.7588

Konecny T, Park JY, Somers KR, Konecny D, Orban M, Soucek F, Parker KO, Scanlon PD, Asirvatham SJ, Brady PA, and

Peer) reviewing PDF | (2020:02:46024:4:0:NEW 3 Oct 2020) 
480

481

482

483

484

485

486

487

488

489

490

491

492

493

494

495

496

497

498

499

500

501

502

503

504

505

506

507

508

509

510

511

512

513

514

515

516

517

518

519

520

Rihal CS. 2014. Relation of chronic obstructive pulmonary disease to atrial and ventricular arrhythmias. Am J Cardiol 114:272-277. 10.1016/j.amjcard.2014.04.030

Koskela J, Kilpeläinen M, Kupiainen H, Mazur W, Sintonen H, Boezen M, Lindqvist A, Postma D, and Laitinen T. 2014. Co-morbidities are the key nominators of the health related quality of life in mild and moderate COPD. BMC Pulm Med 14. 10.1186/1471-2466-14-102

Lainscak M, Von Haehling S, Doehner W, Sarc I, Jeric T, Ziherl K, Kosnik M, Suskovic S, and Anker SD. 2009. Chronic heart failure in patients with acute exacerbation of chronic obstructive pulmonary disease: Prevalence, clinical characteristics, treatment and mortality. J Card Fail 15:S99. 10.1016/j.cardfail.2009.06.113

Liao KM, and Chen CY. 2017. The incidence and risk factors of atrial fibrillation in asian chronic obstructive pulmonary disease patients. Respirology 22:101. 10.1111/resp.13207_42

Liberati A, Altman DG, Tetzlaff J, Mulrow C, Gøtzsche PC, loannidis JP, Clarke M, Devereaux PJ, Kleijnen J, and Moher D. 2009. The PRISMA statement for reporting systematic reviews and meta-analyses of studies that evaluate health care interventions: explanation and elaboration. PLoS Med 6:e1000100. 10.1371/journal.pmed.1000100

Liu J, Wang D, Xiong Y, Liu B, Lin J, Zhang S, Wu B, Wei C, and Liu M. 2017. Association between Coagulation Function and Cerebral Microbleeds in Ischemic Stroke Patients with Atrial Fibrillation and/or Rheumatic Heart Disease. Aging Dis 8:131-135. 10.14336/ad.2016.0715

Lo CK, Mertz D, and Loeb M. 2014. Newcastle-Ottawa Scale: comparing reviewers' to authors' assessments. BMC Med Res Methodol 14:45. 10.1186/1471-2288-14-45

Lopez CM, and House-Fancher MA. 2005. Management of atrial fibrillation in patients with chronic obstructive pulmonary disease. J Cardiovasc Nurs 20:133-140. 10.1097/00005082-200503000-00009

Méndez-Bailón M, Lopez-de-Andrés A, de Miguel-Diez J, de Miguel-Yanes JM, Hernández-Barrera V, Muñoz-Rivas N, Lorenzo-Villalba N, and Jiménez-García R. 2017. Chronic obstructive pulmonary disease predicts higher incidence and in hospital mortality for atrial fibrillation. An observational study using hospital discharge data in Spain (2004-2013). Int J Cardiol 236:209-215. 10.1016/j.ijcard.2017.02.017

Nadeem R, Sharieff A, Tanna S, Sidhu H, Molnar J, and Nadeem A. 2015. Potential Augmentation of the Risk of Ischemic Cerebrovascular Accident by Chronic Obstructive Pulmonary Disease in Patients with Atrial Fibrillation. J Stroke Cerebrovasc Dis 24:1893-1896. 10.1016/j.jstrokecerebrovasdis.2015.04.034

Rusinowicz T, Zielonka TM, and Zycinska K. 2017. Cardiac Arrhythmias in Patients with Exacerbation of COPD. In: Pokorski M, ed. Clinical Management of Pulmonary Disorders and Diseases, 53-62.

Schnabel RB, Sullivan LM, Levy D, Pencina MJ, Massaro JM, D'Agostino RB, Sr., Newton-Cheh C, Yamamoto JF, Magnani JW, Tadros TM, Kannel WB, Wang TJ, Ellinor PT, Wolf PA, Vasan RS, and Benjamin EJ. 2009. Development of a risk score for atrial fibrillation (Framingham Heart Study): a community-based cohort study. Lancet 373:739-745. 10.1016/s0140-6736(09)60443-8

Short PM, Chalmers JD, Akram AR, Singanayagam A, Schembri S, and Williamson PA. 2012. Impact of tachycardia and new onset atrial fibrillation in acute exacerbations of COPD. Thorax 67:A158-A159. 10.1136/thoraxjnl2012-202678.277

Sidney S, Sorel M, Quesenberry CP, Jr., DeLuise C, Lanes S, and Eisner MD. 2005. COPD and incident cardiovascular disease hospitalizations and mortality: Kaiser Permanente Medical Care Program. Chest 128:2068-2075. 10.1378/chest.128.4.2068

Tomioka T, Fukui K, Tanaka S, Ito Y, Shioiri H, Koyama J, and Inoue K. 2019. Influence of atrial fibrillation on cardiac

Peer) reviewing PDF | (2020:02:46024:4:0:NEW 3 Oct 2020) 
521

522

523

524

525

526

527

528

529

530

531

532

533

534

535

536

537

538

539

540

prognosis in chronic obstructive pulmonary disease. Indian Heart J 71:7-11. 10.1016/j.ihj.2018.11.009

Vestbo J, Hurd SS, Agustí AG, Jones PW, Vogelmeier C, Anzueto A, Barnes PJ, Fabbri LM, Martinez FJ, Nishimura M, Stockley RA, Sin DD, and Rodriguez-Roisin R. 2013. Global strategy for the diagnosis, management, and prevention of chronic obstructive pulmonary disease: GOLD executive summary. Am J Respir Crit Care Med 187:347-365. 10.1164/rccm.201204-0596PP

Volchkova EAV, Zateyshchikova AAZ, Shavrin IVS, Zotova IVZ, Nikitin AGN, Brovkin KAB, Nosikov VVN, and Zateyshchikov DAZ. 2015. Polymorphism of gene interleukin- 6 is associated with atrial fibrillation in patients with COPD. Europace 17:iii255. 10.1093/europace/euv182

Wang X, Chen Y, Yao L, Zhou Q, Wu Q, Estill J, Wang Q, Yang K, and Norris SL. 2018a. Reporting of declarations and conflicts of interest in WHO guidelines can be further improved. J Clin Epidemiol 98:1-8. 10.1016/j.jclinepi.2017.12.021

Wang Y-H, Lai C-C, Wang C-Y, Wang H-C, Yu C-J, Chen L, and Taiwan Clinical Trial Consortium R. 2018b. Risks of Pneumonia in COPD Patients with New-Onset Atrial Fibrillation. J Clin Med 7. 10.3390/jcm7090229

Warnier MJ, De Bruin ML, Rutten FH, Hoes AW, and De Boer T. 2010. Drug-induced ECG abnormalities in patients with COPD. Pharmacoepidemiology and Drug Safety 19:S65. 10.1002/pds.2019

Wilchesky M, Ernst P, Brophy JM, Platt RW, and Suissa S. 2012. Bronchodilator use and the risk of arrhythmia in COPD. Part 2: Reassessment in the larger Quebec cohort. Chest 142:305-311. 10.1378/chest.11-1597

Xiao X, Han H, Wu C, He Q, Ruan Y, Zhai Y, Gao Y, Zhao X, and He J. 2019a. Prevalence of Atrial Fibrillation in Hospital Encounters With End-Stage COPD on Home Oxygen: National Trends in the United States. Chest 155:918-927. 10.1016/j.chest.2018.12.021

Peer) reviewing PDF | (2020:02:46024:4:0:NEW 3 Oct 2020) 


\section{Table $\mathbf{1}$ (on next page)}

\section{Characteristics of Included Studies}

Abbreviations: COPD, Chronic obstructive pulmonary disease; AF, Atrial fibrillation; GOLD, The Global Initiative for Chronic Obstructive Lung Disease; ICD-9-CM codes, the International Classification of DiseasesNinth Revision-Clinical Modification; CCS codes, the Clinical Classifications Software; ECG, electrocardiogram; NA, not available.

a: The Newcastle-Ottawa Scale (NOS) assesses the quality of case-control and cohort studies based on categories of selection, comparability, and outcome (or exposure for case-control). NOS uses a star-based system where more stars represent higher quality within a specific category. Studies are awarded a maximum of four stars (****) for selection, two stars (**) for comparability, and three stars $\left({ }^{* * *}\right)$ for outcome.

b: "Education" refers to the completion of high school education.

c: The health related quality of life (HRQOL) was assessed using the self-administered Airways Questionnaire 20 (AQ20). Low HRQoL is defined as AQ20 summary score $\geq 14$. 
Table 1. Characteristics of Included Studies

\begin{tabular}{|c|c|c|c|c|c|c|c|c|c|c|c|c|c|}
\hline \multirow{2}{*}{ Author } & \multirow{2}{*}{ Year } & \multirow{2}{*}{ Country } & \multirow{2}{*}{ Study Design } & \multirow{2}{*}{$\begin{array}{l}\text { Study } \\
\text { Period } \\
\text { (Year) }\end{array}$} & \multirow{2}{*}{$\begin{array}{c}\text { Study } \\
\text { Population }\end{array}$} & \multirow{2}{*}{$\begin{array}{l}\text { COPD } \\
\text { with } \mathrm{AF}\end{array}$} & \multirow{2}{*}{$\begin{array}{c}\text { Age } \\
\text { (years) }\end{array}$} & \multirow{2}{*}{\multicolumn{2}{|c|}{$\begin{array}{c}\text { Identification } \\
\text { of Atrial } \\
\text { Fibrillation }\end{array}$}} & \multirow{2}{*}{ Adjusted variables in logistic regression model } & \multicolumn{3}{|c|}{ Newcastle-Ottawa Scale a } \\
\hline & & & & & & & & & & & Selection & Comparability & Outcome \\
\hline $\begin{array}{r}\text { Rupak } \\
\text { Desai }\end{array}$ & 2019 & USA & $\begin{array}{c}\text { Retrospective } \\
\text { cohort }\end{array}$ & 2010-2014 & 6480799 & 4767401 & 75.8 & CCS code 127 & CCS code 106 & $\begin{array}{l}\text { age, sex, race, admission type, median household } \\
\text { income, length of stay, insurance payer, hospital bed } \\
\text { size, ownership and location/teaching status of the } \\
\text { hospital and all baseline comorbidities }\end{array}$ & $* * * *$ & ** & $* * *$ \\
\hline $\begin{array}{c}\text { Xiaochun } \\
\text { Xiao }\end{array}$ & 2019 & USA & $\begin{array}{c}\text { Retrospective } \\
\text { cohort }\end{array}$ & 2003-2014 & 1345270 & 244488 & 75.5 & $\begin{array}{l}\text { ICD-9 codes } \\
\text { 490.x, 491.x, } \\
\text { 492.x, 494.x, } \\
\text { and 496.x. }\end{array}$ & $\begin{array}{l}\text { ICD-9 code } \\
427\end{array}$ & $\begin{array}{l}\text { age, sex, race, urban living, education b }{ }^{\mathrm{b}} \text {, congestive } \\
\text { heart failure, hypertension, diabetes mellitus, sepsis, } \\
\text { acute respiratory failure, invasive mechanical } \\
\text { ventilation, noninvasive mechanical ventilation, } \\
\text { acute kidney injury and thromboembolism }\end{array}$ & $* * * *$ & $* *$ & $* * *$ \\
\hline $\begin{array}{l}\text { Tomoko } \\
\text { Tomioka }\end{array}$ & 2019 & Japan & Case-control & 2010-2013 & 190 & 42 & 77.5 & GOLD & ECG review & $\begin{array}{l}\text { age, brain natriuretic peptide level, the left atrial } \\
\text { dimension and congestive heart failure }\end{array}$ & $* * * *$ & $* *$ & $* * *$ \\
\hline $\begin{array}{l}\text { Ya-Hui } \\
\text { Wang }\end{array}$ & 2018 & China & $\begin{array}{c}\text { Retrospective } \\
\text { cohort }\end{array}$ & 2000-2011 & 12428 & 6219 & 71.2 & $\begin{array}{l}\text { ICD-9 codes } \\
491,492,496\end{array}$ & $\begin{array}{l}\text { ICD-9 code } \\
427.31\end{array}$ & $\begin{array}{l}\text { age, sex, index year of AF, monthly income, hospital } \\
\text { level, severe exacerbation of COPD in one year } \\
\text { prior to index date (never, } 1 \text {, or } \geq 2 \text { times/year), } \\
\text { medications for COPD, medications for } \\
\text { hypertension, other medications and individual } \\
\text { comorbidities }\end{array}$ & **** & $* *$ & $* * *$ \\
\hline
\end{tabular}




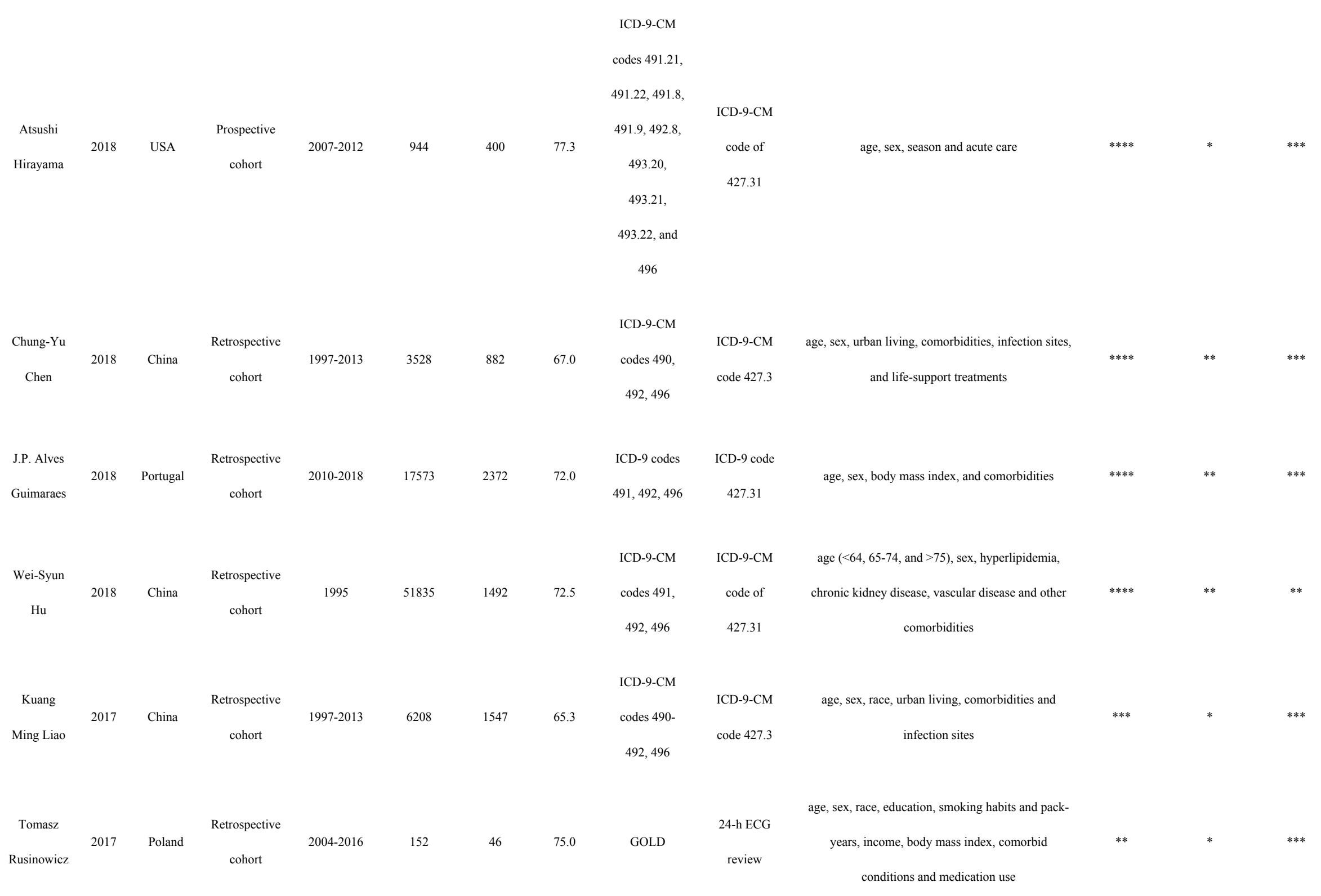




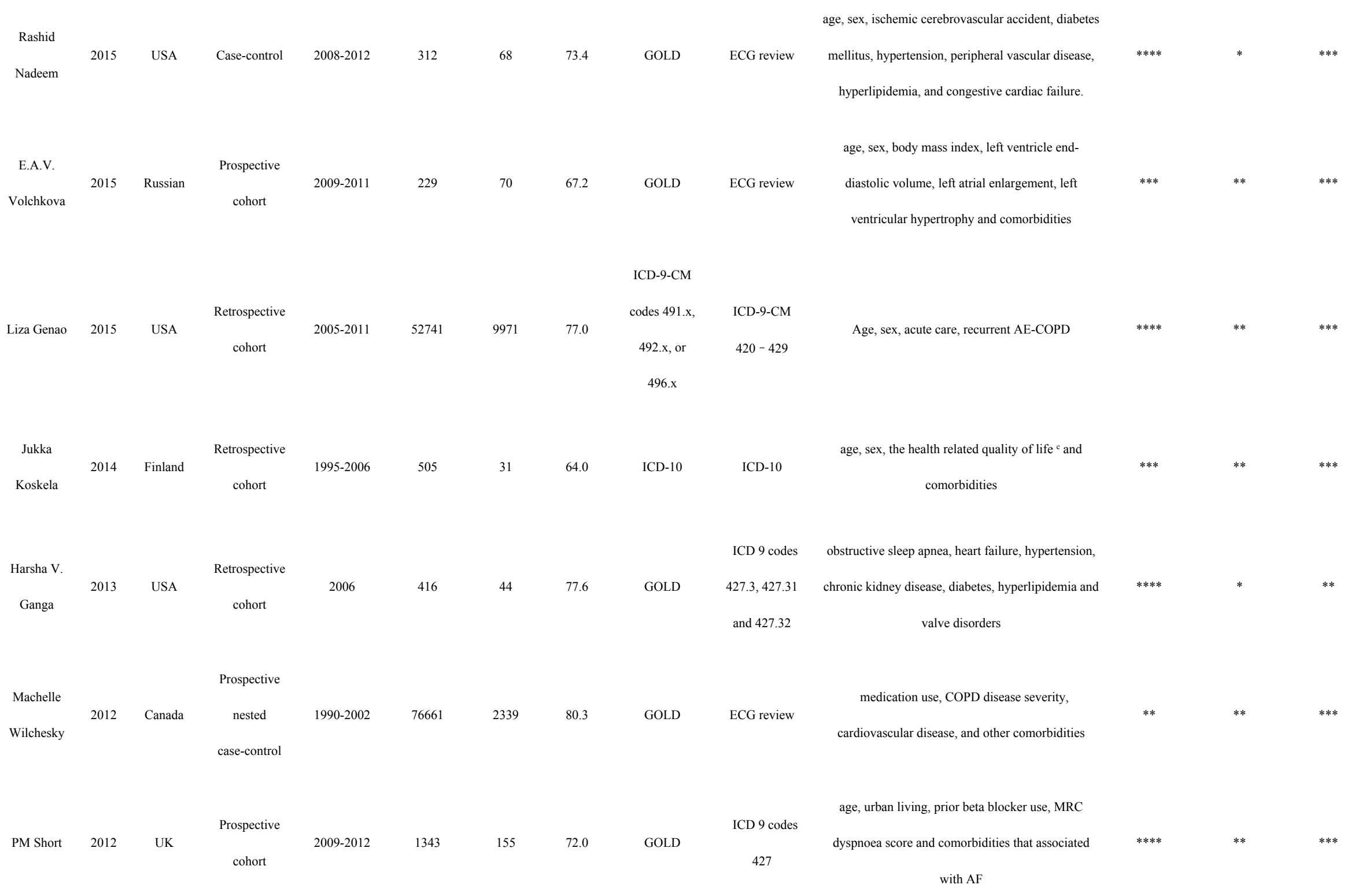




\begin{tabular}{|c|c|c|c|c|c|c|c|c|c|c|c|c|c|}
\hline Bartolome & & & & & & & & & & age, sex, urban living, tiotropium use, comorbidities & & & \\
\hline Celli & 2010 & Germany & Case-control & 2008 & 19545 & 306 & 65.0 & GOLD & ECG review & and smoking habits and pack-years & $* * * *$ & ** & *** \\
\hline Miriam J & & Netherla & Retrospective & & & & & & 12-lead ECG & age, sex, diabetes mellitus, myocardial infarction, & & & \\
\hline & 2010 & nds & & 2009 & 404 & 22 & 72.9 & GOLD & & & $* * * *$ & ** & *** \\
\hline Mitja & & & Retrospective & & & & & & & & & & \\
\hline Lainscak & 2009 & Germany & cohort & 2002-2007 & 960 & 288 & 71.0 & ICD-10 & ICD-10 & age, sex, tobacco consumption and comorbidities & $* * *$ & ** & $* * *$ \\
\hline
\end{tabular}

2 Abbreviations: COPD, Chronic obstructive pulmonary disease; AF, Atrial fibrillation; GOLD, The Global Initiative for Chronic Obstructive Lung Disease; ICD-9-CM codes, the International Classification of Diseases-Ninth Revision-Clinical Modification; CCS codes, the Clinical

Software; $\quad$ ECG, electrocardiogram;

NA,

not

available.

5 a: The Newcastle-Ottawa Scale (NOS) assesses the quality of case-control and cohort studies based on categories of selection, comparability, and

6 outcome (or exposure for case-control). NOS uses a star-based system where more stars represent higher quality within a specific category. Studies

7 are awarded a maximum of four stars $(* * *)$ for selection, two stars $(* *)$ for comparability, and three stars $(* * *)$ for outcome.

8 b: "Education" refers to the completion of high school education.

9 c: The health related quality of life (HRQoL) was assessed using the self-administered Airways Questionnaire 20 (AQ20). Low HRQoL is

10 defined as AQ20 summary score $\geq 14$. 


\section{Table 2 (on next page)}

Comparison of risk factors for COPD-associated AF and Non-COPD-associated AF

Abbreviations: COPD, Chronic obstructive pulmonary disease; AF, Atrial fibrillation; DM, Diabetes mellitus; MI, Myocardial infarction; CAD, Coronary artery disease; HF, Heart failure; NA, not available.a. Odds ratio $(95 \% \mathrm{Cl})$; b. Hazard ratio $(95 \% \mathrm{Cl})$.Shown are effect sizes for factors evaluated in current study (COPD-associated AF) and by Schnabel et al (Schnabel et al. 2009) and Chamberlain et al (Chamberlain et al. 2011) (non-COPD-associated AF) that are similarly defined. 
1 Table 2. Comparison of risk factors for COPD-associated AF and Non-COPD-associated AF

\begin{tabular}{|c|c|c|c|}
\hline \multirow[b]{3}{*}{ Risk Factors } & \multicolumn{3}{|c|}{ Effect Size } \\
\hline & \multirow[b]{2}{*}{ COPD-Associated AF a } & \multicolumn{2}{|c|}{ Non-COPD-Associated AF } \\
\hline & & Schnabel et al ${ }^{b}$ & Chamberlain et al ${ }^{b}$ \\
\hline Black race (vs White) & $0.51(0.45-0.59)$ & $0.53(0.44-0.63)$ & $0.52(0.43-0.62)$ \\
\hline Male (vs Female) & $1.26(1.20-1.34)$ & $1.90(1.58-2.29)$ & $1.92(1.60-2.30)$ \\
\hline Age $>65(\mathrm{vs} \leq 65)$ & $3.29(3.25-3.33)$ & $2.28(2.08-2.49)$ & NA \\
\hline $\mathrm{DM}$ & $0.89(0.54-1.49)$ & $1.10(0.87-1.38)$ & $1.87(1.51-2.32)$ \\
\hline Prior beta blocker use & $0.68(0.35-1.28)$ & $1.80(1.48-2.18)$ & $2.55(2.13-3.04)$ \\
\hline Hypertension & $0.95(0.70-1.28)$ & NA & $2.16(1.67-2.79)$ \\
\hline MI & $1.41(1.19-1.69)$ & $1.34(1.02-2.03)$ & NA \\
\hline CAD & $2.57(1.01-6.49)$ & NA & $2.21(1.71-2.84)$ \\
\hline $\mathrm{HF}$ & $3.49(2.70-4.52)$ & $3.20(1.99-5.16)$ & $3.03(2.32-3.95)$ \\
\hline Left ventricular hypertrophy & $2.23(1.12-4.34)$ & $1.36(1.03-1.80)$ & NA \\
\hline
\end{tabular}

2 Abbreviations: COPD, Chronic obstructive pulmonary disease; AF, Atrial fibrillation; DM, Diabetes mellitus;

3 MI, Myocardial infarction; CAD, Coronary artery disease; HF, Heart failure; NA, not available.a. Odds ratio 4 (95\% CI); b. Hazard ratio $(95 \% \mathrm{CI})$. Shown are effect sizes for factors evaluated in current study (COPD- 
5 associated AF) and by Schnabel et al (Schnabel et al. 2009) and Chamberlain et al (Chamberlain et al. 2011)

6 (non-COPD-associated AF) that are similarly defined. 
Figure 1

\section{PRISMA (preferred reporting items for systematic reviews and meta-analyses) flow diagram and exclusion criteria}
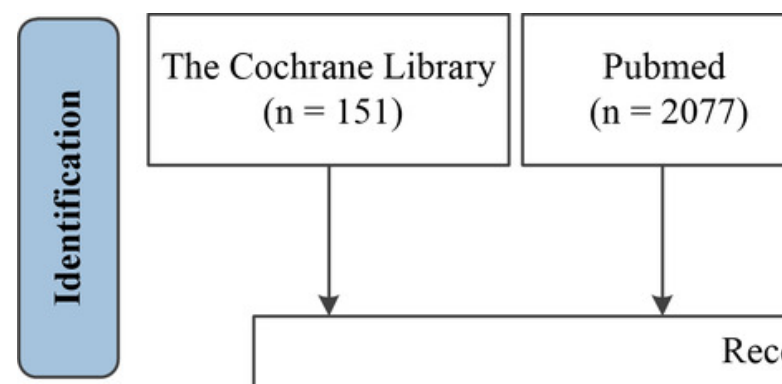

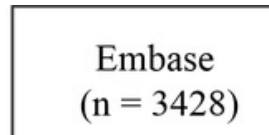

$(\mathrm{n}=3428)$

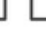

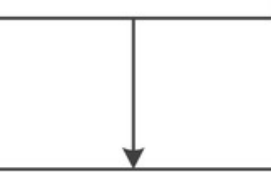
Web of Science
$(\mathrm{n}=1059)$

Additional records identified through other sources $(\mathrm{n}=2)$

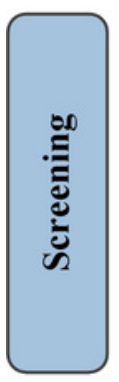

Records after duplicates removed

$(\mathrm{n}=5522)$
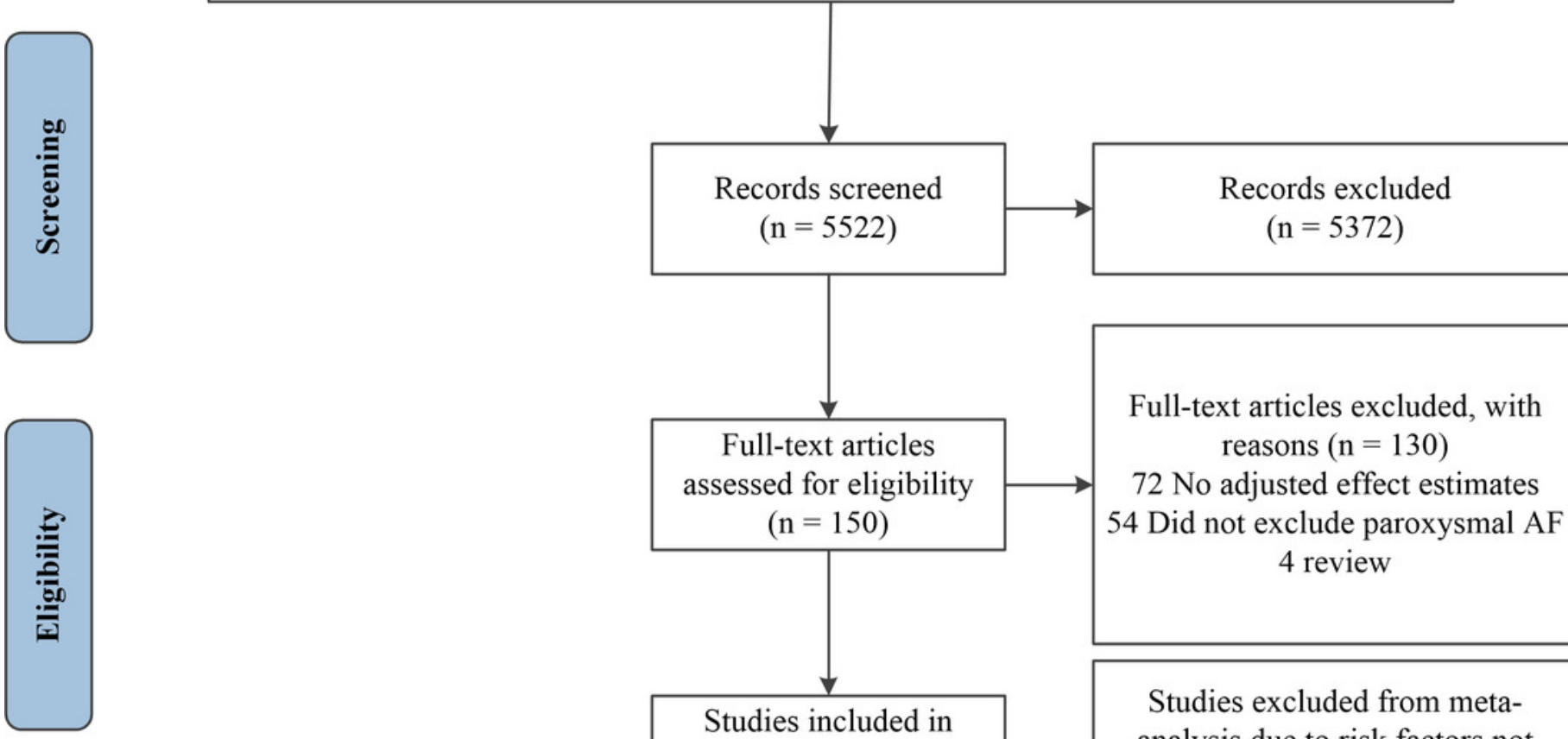

Full-text articles assessed for eligibility $(\mathrm{n}=150)$
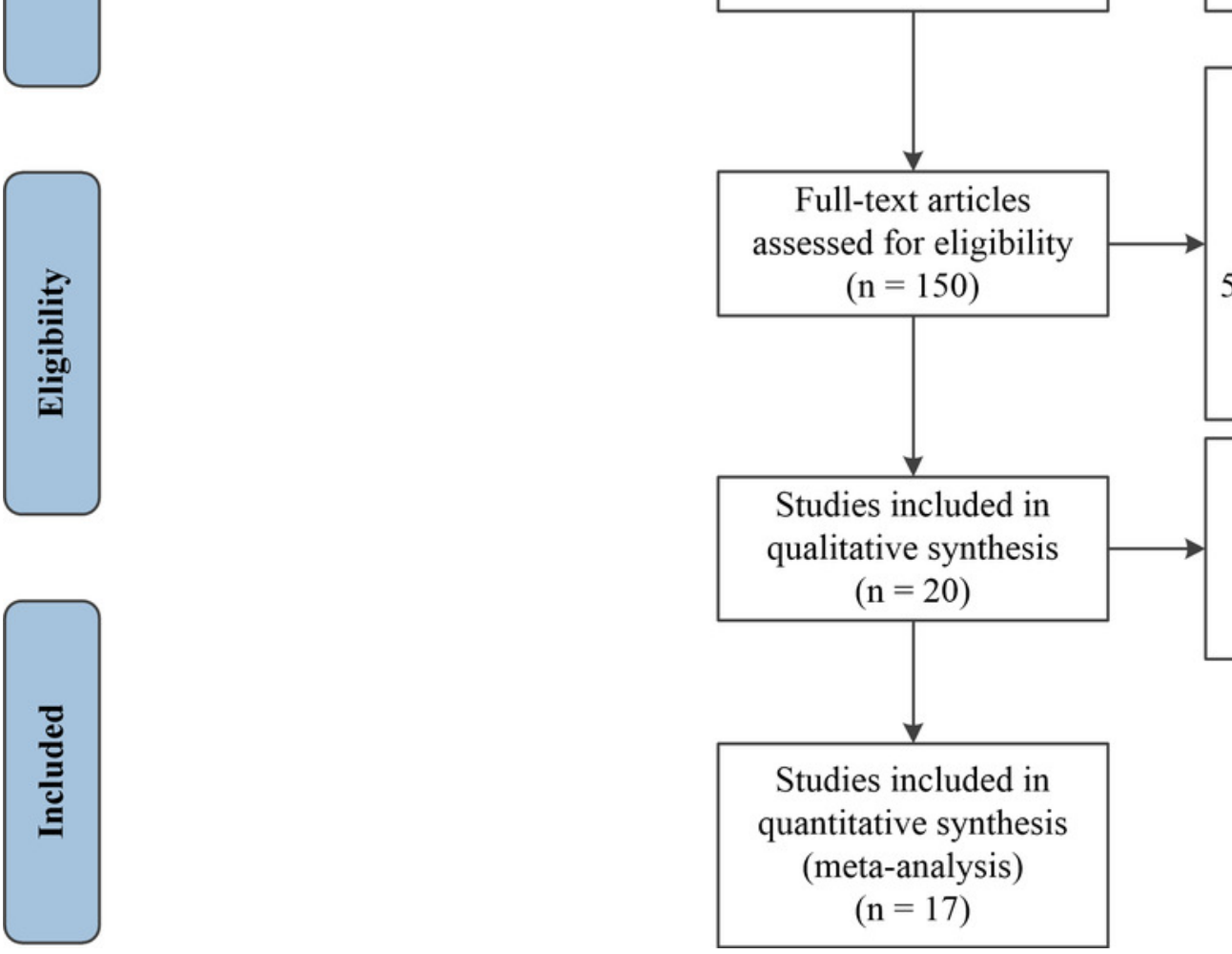

Studies excluded from metaanalysis due to risk factors not reported in two or more studies 


\section{Figure 2}

Risk factors for AF in COPD patients stratified by factor type and pooled versus unpooled analysis.

Filled diamonds show the pooled-adjusted odds ratios (OR) for risk factors from the metaanalysis and the adjusted OR for un-pooled risk factors. Error bars denote $95 \% \mathrm{Cls}$.

Abbreviations: COPD, Chronic obstructive pulmonary disease; AF, Atrial fibrillation; OR, Odds ratio; $95 \% \mathrm{Cl}$, Confidence intervals; IMV, Invasive mechanical ventilation; NMV, Noninvasive mechanical ventilation; MI, Myocardial infarction; CAD, Coronary artery disease; $H F$, Heart failure; CHF, Congestive heart failure; PVD, Peripheral vascular disease; DM, Diabetes mellitus; HRQoL, the Health related quality of life; AKI, Acute kidney injury; CKD, Chronic kidney disease; OSA, Obstructive sleep apnea.* Below high school degree (vs High school degree or above).\# The HRQoL was assessed using the self-administered Airways Questionnaire 20 (AQ20). Low HRQoL is defined as AQ20 summary score $\geq 14$. 


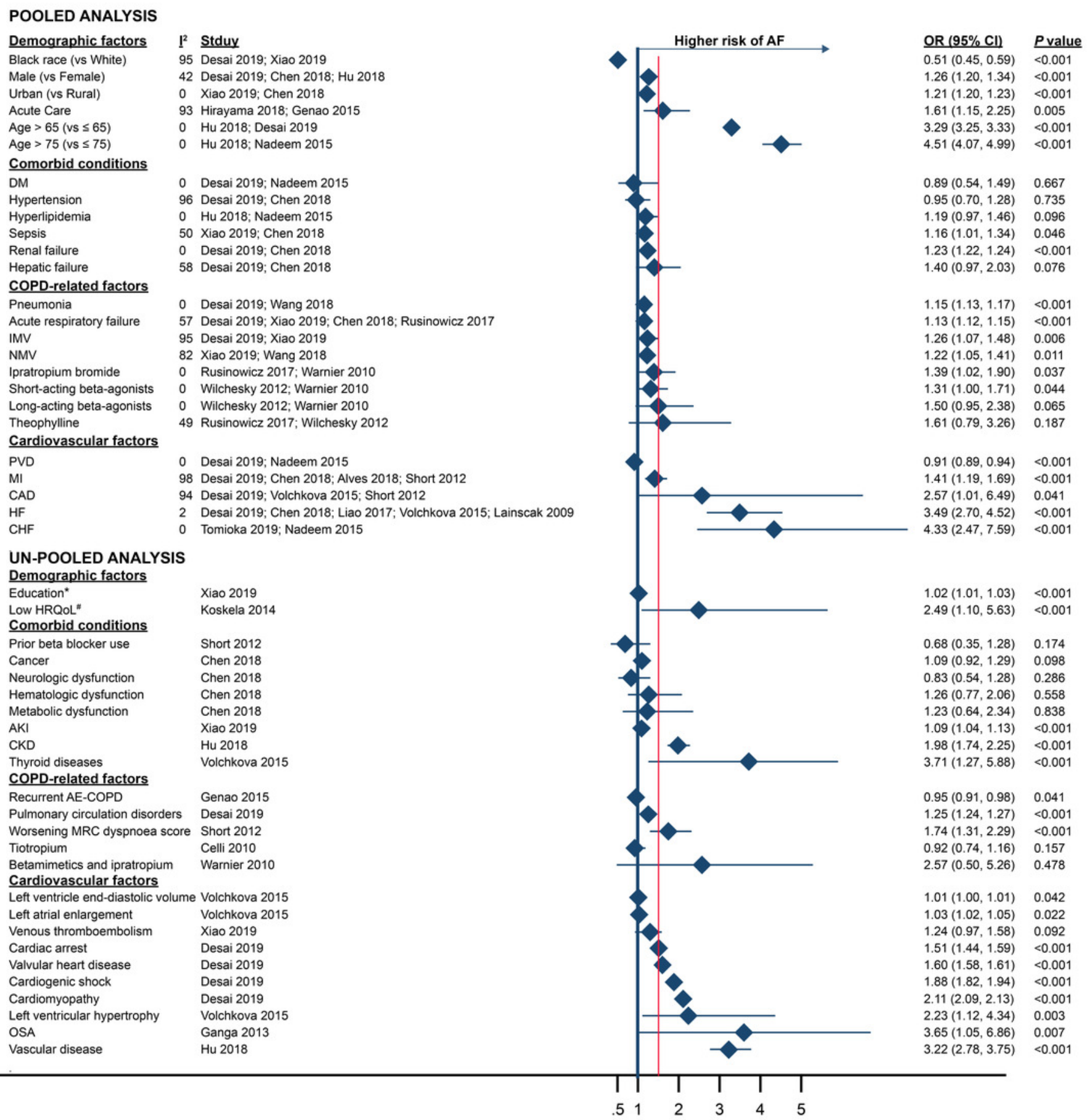

